\title{
LA EFECTIVIDAD DE UN DISEÑO CALL PARA EL APRENDIZAJE DEL RESUMEN EN ESCOLARES*
}

\section{Constance Silvia Gómez Veloso**}

\begin{abstract}
Resumen
Este artículo se enmarca en el ámbito de la Lingüística Aplicada en la interdisciplina de CALL (Computer Assisted Language Learning, en español, Aprendizaje de Lenguas Asistida por Computador), específicamente en el aprendizaje de un aspecto lingüístico de la lengua materna: el resumen de un texto informativo de alumnos de sexto año básico. El objetivo de este estudio es desarrollar la competencia en la producción escrita de un resumen utilizando un diseño CALL basado en un Enfoque Colaborativo y en un Enfoque por Tareas, mediado por el uso de Google Docs y Blog. Con el objeto de corroborar la efectividad del diseño se desarrolló un estudio cuasi-experimental, longitudinal con pre-postest y grupo de control. Finalmente se muestran los resultados de esta investigación, los que demuestran que un diseño CALL mejora la producción de resúmenes en estudiantes de sexto básico.
\end{abstract}

Palabras clave: CALL, resumen, enfoque colaborativo, enfoque por tareas.

\section{THE EFFECTIVENESS OF A DESIGN FOR LEARNING CALL SUMMARY IN SCHOOL}

\begin{abstract}
This article falls within the field of Applied Linguistics at the interdisciplinary of CALL (Computer Assisted Language Learning, in Spanish, Aprendizaje de Lenguas Asistido por Computador), specifically in learning a linguistic aspect of language: a summary of a informative text basic sixth graders. The aim of this study is to develop proficiency in the written production of a summary using a design-based CALL Collaborative Approach and Approach by Tasks, mediated by the use of Google Docs and Blog. In order to corroborate the effectiveness of the design was developed a quasi-experimental pre-posttest longitudinal and control group. Finally shows the results of this investigation show that the design improves production CALL abstracts sixth grade students.
\end{abstract}

Keywords: CALL, summary, collaborative approach, task-based approach.

* Artículo asociado al proyecto de investigación "Producción de resúmenes de textos informativos con herramientas de la web 2.0 mediante el uso de principios de CALL aplicados a la lengua materna". Tesis para optar al grado de Magíster en Lingüística aplicada, Universidad de Concepción.

** Chilena, Profesora de Español, Diplomada en Informática Educativa y Gestión del Conocimiento, Candidata a Magíster en Lingüística Aplicada, Becaria CONICYT. Universidad de Concepción, Concepción, Chile. constancegomez@udec.cl 


\section{Introducción}

El resumen se define como un tipo de discurso que proporciona una macroestructura general del discurso que resume, es decir, un texto que incluye la macroestructura que da cuenta de lo que trata el texto al que se refiere, lograda, a su vez, por la aplicación de una serie de macro-reglas que no se aplican de forma fortuita, sino que están restringidas por los conocimientos, propósitos, y otras condicionantes impuestas por quien las produce (Van Dijk, 1980). Sin embargo, el resumen, si bien implica transformaciones (brevedad, condensación, eliminación), a pesar de todas las variables involucradas, tiene que guardar con el texto base una relación que preserve su contenido genuino (Bustamante y Jurado, 1997).

El objetivo general de este estudio es desarrollar la habilidad de producción de resúmenes de un texto informativo, en la lengua materna, mediante un diseño CALL que combina un Enfoque Colaborativo con un Enfoque por Tareas para mediar el uso de herramientas de la web 2.0. Este diseño pretende resolver el siguiente problema de investigación: cómo mejorar la producción de resúmenes en alumnos de sexto básico.

Dada la necesidad y las potencialidades de las aplicaciones computacionales, se selecciona CALL como un diseño que abarca el problema en toda su complejidad: habilidad lingüística, el uso y diseño de materiales y tareas efectivas.

El interés de los investigadores por el Enfoque Colaborativo y el Enfoque por Tareas se fundamenta en que estos dos enfoques metodológicos tienen, como uno de sus objetivos, capacitar al estudiante para desarrollar y mejorar sus habilidades comunicativas. Junto con lo anterior, estos enfoques responden a una necesidad actual, ya que con sus principios se acercan a la nueva forma en que los estudiantes de hoy aprenden.

\section{Marco Teórico}

\section{Producción Textual}

Existen investigaciones que muestran efectos positivos en el trabajo de la escritura en CALL, en términos de: actitudes del escritor, la extensión del texto, su calidad y cantidad y, en algunos casos, la calidad de las revisiones (Hubbard, 2009, Pennington, 2004). 
Cuando analizamos la escritura de un resumen, resulta importante comprender su estructura interna. Los marcadores textuales son elementos centrales en la construcción de este tipo de textos, ya que "señalan los accidentes de la prosa: la estructura, las conexiones entre frases, la función de un fragmento, etc. Tienen forma de conjunciones, adverbios, locuciones conjuntivas o incluso sintagmas, y son útiles para ayudar al lector a comprender el texto" (Cassany, 2004: 82).

Cassany (2004) señala que existen marcadores textuales que sirven para:

Estructurar el texto: afectan a un fragmento relativamente extenso de texto (párrafo, apartado, grupo de oraciones...). Éstos, además, tienen su utilidad para establecer orden y relaciones significativas entre frases.

Ejemplos: los que indican tiempo, espacio y los que están para: introducir el tema del texto, iniciar un tema nuevo, marcar orden, distinguir, continuar sobre el mismo punto, hacer hincapié, detallar, resumir y acabar.

Estructurar ideas: afectan a fragmentos más breves de texto (oraciones, frases...) y conectan las ideas entre sí en el interior de la oración. Son las conjunciones de la gramática tradicional.

Ejemplos: los que indican: causa, consecuencia, condición, finalidad, oposición y objeción.

Los marcadores textuales se deben colocar en posiciones importantes dentro del texto, de modo que el lector los distinga solo con observarlo, incluso antes de empezar a leer, y de esa forma, se pueda hacer una idea de la organización del texto. No se debe abusar de ellos porque pueden atiborrar la prosa (Cassany, 2004).

Cassany señala que la escritura cuenta con su propia organización jerárquica, la que permite articular cualquier mensaje por apartados. Esto ocurre ya que la organización de las ideas debe quedar reflejada en el texto de alguna forma, si se pretende que el lector siga la estructura que se le ha dado al mensaje. De acuerdo a los apartados que este autor propone, esta investigación se centró en el nivel de la frase.

A continuación en el Esquema 1 observaremos la organización jerárquica de todo texto. 


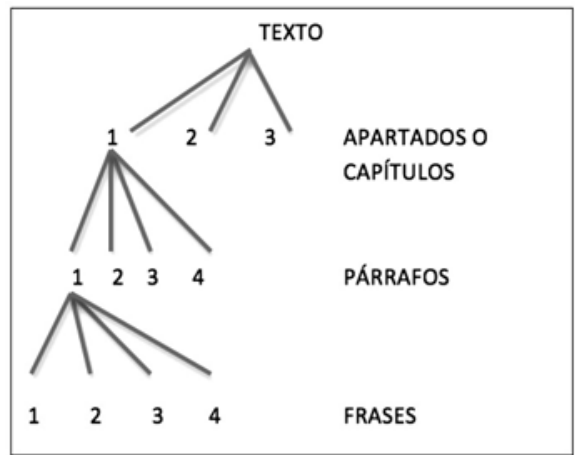

Esquema 1: Estructura de un texto (Cassany, 2004)

\section{El resumen}

Frente a la pregunta ¿Qué es un resumen?, tanto la definición, como los procedimientos que orientan el cómo se hace y las relaciones que mantiene con la comprensión de un texto, con la memoria, y con la captación de la información son expuestos desde diferentes dominios teóricos (Bustamante y Jurado, 1997). Cuando se realiza por escrito, el resumen es una reescritura de un texto que debe cumplir con dos requisitos para mantener la equivalencia informativa: representar el pensamiento y los principales contenidos propuestos por el autor y economizar medios; es decir, presentar la misma información del texto original, pero con un número menor de palabras, lo cual implica eliminar información redundante o secundaria (Condemarín, 1998).

Venegas (2010) añade un factor importante en la producción del resumen, ya que no solo lo define como una técnica de producción, sino también como una técnica evaluativa. Es así como se menciona que el resumen no debe resultar una mera reescritura del texto original, como señala Condemarín (1998), sino una reelaboración de lo que el texto propone globalmente. En este sentido, el resumen es la textualización de un significado que representa de modo abstracto el significado total del contenido de una lectura, también llamado macrosemantización.

Según Condemarín la calidad de un resumen depende principalmente de cómo haya sido leído el texto que se quiere resumir. En tal sentido, plantea que es necesario diferenciar la lectura de un texto narrativo de uno informativo. En el caso de los textos narrativos, será importante 
hacerse una idea clara del carácter de los personajes, sus motivaciones y la situación en la cual ocurren los hechos. Por el contrario, en el caso de un texto informativo, el lector deberá detectar las palabras claves y las frases que le permitirán resumir los planteamientos del autor. El significado de un texto narrativo, puede tomar forma al final del texto; el significado de un texto informativo se construye a lo largo de toda la lectura. Por estas razones es importante estimular a los estudiantes a realizar esta tarea de acuerdo a las siguientes consideraciones: tomar notas y subrayar, identificar las ideas principales y secundarias, diferenciar los conceptos o las ideas relevantes de los que no lo son, reparar en los detalles que apoyan o sustentan los conceptos o las ideas importantes, percibir la relación entre los distintos planteamientos, determinar qué vale o no la pena de analizar, retener o registrar.

De acuerdo con las consideraciones que se debe tener en la producción de un resumen, mencionadas anteriormente, la identificación del tema y de las ideas fundamentales contenidas en un texto dan una base importante para resumir. Pero éste -el resumen- requiere de una concreción, de una forma escrita y de un sistema de relaciones que en general no se desprende directamente de la identificación o construcción de las ideas principales (Solé, 1996).

Se advierte que en torno a la enseñanza del resumen existen opiniones contradictorias, puntos de vistas encontrados y que no siempre resulta fácil tomar una opción, parte de una concepción de la lectura y de las estrategias que permiten hacerla comprensiva y eficaz que no la considera como un compendio de sus habilidades. A leer se aprende leyendo, y a resumir, resumiendo.

\section{CALL: El Aprendizaje de Lenguas Asistido por Computador}

El Aprendizaje de Lenguas Asistido por Computador (CALL) se puede definir como la búsqueda y el estudio de las aplicaciones del computador en la enseñanza y el aprendizaje (Levy, 2006). En general, el objetivo principal de CALL es mejorar la capacidad de aprendizaje de aquellos que están aprendiendo un idioma a través de medios informáticos (Cameron, 1999).

Las investigaciones realizadas en CALL han demostrado que el uso de una aplicación computacional facilita e incentiva el aprendizaje de un idioma (García y Ferreira, 2010). 
El uso del computador reconsidera los roles tradicionales de las partes interesadas: los alumnos, docentes e investigadores, los que han tenido que adaptarse a las exigencias y oportunidades que ofrece la amplia gama de nuevas tecnologías. Con la llegada del computador en red e Internet, los estudiantes son cada vez más llamados a diseñar y ejecutar su propio equipo basado en actividades (Gruba, 2004).

Egbert (2005) menciona que CALL significa que los estudiantes aprenden un idioma, en un contexto a través de las tecnologías informáticas: el lenguaje aprendido puede ser cualquier lengua del mundo.

Esta investigación se basa en la definición de CALL que nos ofrece Hubbard (2009): una definición que señala que el aprendizaje de idiomas asistido por computador es un campo de investigación que estudia cualquier proceso en el que un estudiante aprende utilizando una computadora y, como consecuencia, mejora su lengua. Para este autor, aprender una lengua en sus aspectos formales siempre es un proceso cognitivo complejo, por ende, desarrollar un aprendizaje lingüístico en la lengua materna sería un proceso similar a cuando un estudiante aprende una segunda lengua. En definitiva, para Hubbard la lengua materna es como una segunda lengua cuando se aprenden estructuras lingüísticas formales.

Esta definición de Hubbard (2009) nos permite desarrollar dos interrogantes: ¿Qué se entiende por computador? Y ¿qué queremos decir por mejorar?

El primero de ellos, según Hubbard (2009) es una cuestión importante en la definición porque CALL no es simplemente considerar el escritorio y los dispositivos portátiles, como los computadores. También incluye las redes que conectan a los dispositivos periféricos asociados con ellos y una serie de otras innovaciones tecnológicas, tales como PDAs (asistentes personales digitales), reproductores de mp3, teléfonos móviles, pizarras electrónicas, e incluso reproductores de DVD que están dentro las computadoras (Hubbard 2009).

La segunda pregunta, se puede responder con respecto a una serie de perspectivas diferentes: 
La eficiencia del aprendizaje: la capacidad que tienen los estudiantes de recoger el conocimiento lingüístico o habilidades con más o menos esfuerzo.

El aprendizaje eficaz: los estudiantes retienen el conocimiento o habilidad lingüística con el fin de hacer más asociaciones y/o aprender más de lo que necesitan.

Acceso: los estudiantes pueden obtener los materiales o pueden tener la experiencia de interactuar con otros que de otra manera sería difícil o imposible de conseguir o hacer.

Comodidad: los estudiantes pueden estudiar y practicar con la misma eficacia a través de una gama más amplia de tiempos y lugar.

Motivación: Los estudiantes disfrutan más del proceso de aprendizaje de idiomas y así participan más plenamente.

La eficiencia institucional: los estudiantes requieren menos tiempo del profesor, menos recursos y además es menos costoso (Hubbard, 2009).

\section{Diseño CALL}

Existe un marco general en cualquier investigación en CALL, que consiste en 3 módulos, que según Hubbard (1996) son cíclicos: desarrollo, evaluación e implementación.

Módulo de desarrollo: determina los elementos esenciales que intervienen en el desarrollo de un estudio. Está caracterizado por el enfoque de enseñanza de la lengua, el diseño y el procedimiento. El enfoque de enseñanza determina la selección de la tecnología junto con la metodología para desarrollar la lengua y el entorno de aprendizaje. En el diseño se definen los elementos que constituyen un curso de lengua: las metas de aprendizaje, los objetivos, el plan, los métodos y el rol de los agentes; el nivel de aprendizaje, necesidades e intereses, estrategias de aprendizaje. El procedimiento considera la selección del tipo de los materiales y de actividades que realizarán los usuarios.

Módulo de implementación: En el módulo de implementación se encuentran los factores más importantes para que ocurra el aprendizaje en este contexto: el uso de software o plataforma, la accesibilidad, los materiales tutoriales del curso y el enfoque de enseñanza que opera como filtro para estimar el tipo de control que ejerce el profesor sobre las tareas. 
Módulo de evaluación: En este módulo se debe considerar si las actividades y materiales conducen al logro de los objetivos preestablecidos según el enfoque seleccionado. En consecuencia, el enfoque, el diseño y el procedimiento, se evalúan en tanto efectividad para el profesor, para el estudiante y la aplicación.

\section{Enfoques Metodológicos}

\section{Enfoque por Tareas}

Richards \& Rodgers (2004) afirman que el aprendizaje basado en tareas es un enfoque que utiliza la tarea como la unidad principal de la planificación y la enseñanza. Por su parte, Willis \& Willis (2007) señalan que una buena tarea no sólo genera interés y crea un aceptable grado de desafío, sino que también genera oportunidades para que los alumnos experimenten y activen tanto el idioma como sea posible.

Las tareas como unidad lingüística en la vida real se definen como aquellas actividades para las cuales utilizamos la lengua en nuestra vida cotidiana (Long, 1985).

Estaire (2007) señala que existe un marco para la programación de una unidad didáctica mediante tareas: elección del tema, centro de interés o foco de atención, especificación de objetivos a partir del análisis de la tarea final, especificación de contenidos lingüísticos y de otros tipos derivados de la tarea final, planificación del proceso, análisis de toda la secuencia programada y finalmente la programación de los instrumentos y procedimientos de evaluación a utilizar por profesores y estudiantes a lo largo de la unidad, como parte integral del proceso de aprendizaje.

Por otra parte, Nunan (2001) aporta una clasificación de las tareas, las que se dividen en dos grupos: tareas del mundo real y tareas pedagógicas. Las primeras se refieren a un tipo de tarea comunicativa que se logra en el mundo, fuera de la sala de clases. Las segundas son aquellas en las que los estudiantes trabajan en el aula comprendiendo, produciendo o interactuando con la lengua, mientras su atención está principalmente puesta en el significado más que en la forma.

Finalmente, Skehan (2008) sugiere una clasificación distinta a la de Nunan, ya que, según este autor, las tareas se dividen en tres etapas: las pre-tareas, las tareas de proceso y las post-tareas. A pesar de que 
la primera y la tercera fase no son obligatorias, pueden jugar un rol importante en la efectividad de la metodología.

En definitiva, una metodología basada en tareas permite que el estudiante aprenda de una manera más metódica, ordenada y participativa, lo que redunda en una interacción del aprendiente más activa dentro del proceso de aprendizaje, lo que permite que ellos descubran el conocimiento de forma autónoma. De este modo, es el estudiante el responsable de su propio aprendizaje, dejando al docente como un guía hacia el conocimiento.

\section{Enfoque Colaborativo}

Actualmente estamos asistiendo al nacimiento de un nuevo paradigma en la tecnología de la educación: uno que se basa en diferentes hipótesis sobre la naturaleza del aprendizaje y que incorpora un nuevo conjunto de prácticas de investigación (Chapelle, 2001). En este nuevo paradigma, podemos decir que se considera que las personas ya no aprenden de manera aislada, sino en contacto permanente con sus pares.

Gokhale (1995) define al aprendizaje colaborativo como un método de instrucción en el cual los estudiantes trabajan en grupos con un objetivo académico común. Se añade que el término aprendizaje colaborativo se refiere a un método de instrucción en la que estudiantes, de diferentes niveles de rendimiento, trabajan en pequeños grupos hacia una meta común. Los estudiantes son responsables unos de otros del aprendizaje. Por lo tanto, el éxito de un estudiante sirve de ayuda a otros estudiantes para tener éxito.

En otra de sus ventajas, como uno de los postulados constructivistas que concibe a la educación como proceso de socio-construcción, el enfoque colaborativo permite conocer las diferentes perspectivas para abordar un determinado problema, desarrollar tolerancia en torno a la diversidad y reelaborar una alternativa conjunta (Calzadilla, 2001).

Hay que considerar entonces que el entorno de aprendizaje electrónico reúne características que son especialmente poderosas para la colaboración, tales como su: interactividad, ubicuidad y sincronismo. El aprendizaje colaborativo entonces, nace y responde a un nuevo contexto socio cultural donde se define el "cómo aprendemos" (socialmente) y "dónde aprendemos" (en red) (Zañartu, 2008). Es por esto mismo que 
se menciona que la teoría constructivista tendría una actualización o bien, una nueva arista de investigación, llamada conectivismo, que viene a modernizar la idea de que el individuo construye su aprendizaje socialmente. El conectivismo no deja de lado esa idea, solo que actualiza el espacio de aprendizaje, que ya no es solo social, sino conectado entre sus pares.

\section{Web 2.0: herramientas gratuitas para la escritura en colaboración}

La web 2.0 podría definirse como la Red (o Internet) convertida en un espacio social, que da cabida a todos los agentes sociales, capaz de dar soporte y formar parte de una verdadera sociedad de la información, la comunicación y/o el conocimiento (Lázaro, 2009). En la web 2.0 cambia profundamente el rol de los usuarios: de pasivos y receptores a usuarios activos, productores y colaborativos. Esta web se caracteriza por las redes sociales y los espacios de colaboración en los que se desarrolla la llamada inteligencia colectiva (Piscitelli, 2010).

Existen herramientas de la web 2.0 que han sido desarrolladas para promover la escritura en colaboración y en la web: la más reciente de las herramientas gratuitas en línea es Google Docs (http: / / docs.google.com), la que ha sido utilizada por los profesores de idiomas para este propósito. Reconociendo que la escritura auténtica requiere una audiencia, los profesores de idiomas utilizan una variedad de equipos, basados en las opciones para la publicación de trabajos de los estudiantes. Inicialmente, esto se hizo utilizando el software de procesamiento de textos dedicado a crear y dar formato a las publicaciones impresas. En la década de los 90, las páginas web se convirtieron en una opción para que los estudiantes produjeran y, recientemente, los Blogs y los Wikis han abierto nuevas oportunidades para que los estudiantes, particularmente aquellos con menos habilidades técnicas, puedan obtener su producto por escrito frente a una audiencia en línea (Hubbard, 2009).

En este contexto, las TIC (tecnologías de la información y la comunicación) han desempeñado un papel fundamental en la configuración de nuestra sociedad y nuestra cultura (Adell, 2007).

Dentro de este conjunto de procesos nace el paradigma de las nuevas tecnologías, que son las redes informáticas. Los computadores, de forma aislada, ofrecen una gran cantidad de posibilidades, pero conectados 
incrementan su funcionalidad en varios órdenes de magnitud (Adell, 2007).

\section{Diseño de la Investigación}

Este estudio tiene como objetivo general desarrollar la habilidad de producción de resúmenes de un texto informativo en la lengua materna mediante un diseño CALL que combina un Enfoque Colaborativo con un Enfoque por Tareas para mediar el uso de herramientas web 2.0. Este objetivo general surge de la hipótesis que un diseño CALL que combine el Enfoque por Tareas con el Enfoque Colaborativo utilizando herramientas web 2.0 mejora la producción de resúmenes de textos informativos en estudiantes de sexto básico.

La investigación tiene un enfoque metodológico cuantitativo y está basada en un diseño cuasi-experimental longitudinal con pre-post test y grupo control, en el cual se compara el incremento del aprendizaje del resumen obtenido por dos grupos. La primera medición que se aplicó fue un pre-test, el cual se realizó en una etapa inicial, anterior a la intervención lingüística. La segunda se llevó a cabo al finalizar dicha intervención, en la que se les aplicó un post-test. Se utiliza un diseño cuasi-experimental, ya que no hay una asignación aleatoria de los sujetos de ambos grupos y no hay un control efectivo de las variables de selección.

\section{La muestra}

La muestra utilizada en esta investigación corresponde a un muestreo no aleatorio, con grupos naturales. Consiste en 44 estudiantes de un colegio particular subvencionado que cursan sexto básico, divididos en grupo experimental y grupo de control. Cada grupo está compuesto por 22 sujetos: 9 hombres y 11 mujeres en el grupo experimental y 14 hombres y 7 mujeres en el grupo de control. Ambos grupos tienen edades entre 11 y 12 años.

Para fundamentar la selección de ambos grupos, se aplicó una prueba estadística $T$ Student con el fin de comparar tanto los promedios de cada curso como los resultados del pre-test y, de ese modo, comprobar que ambos grupos son de una misma población. Esta prueba se realizó utilizando un programa estadístico llamado STATA 11, aplicación desarrollada para realizar análisis estadísticos. 
Al aplicar esta prueba estadística, con un 95\% de confianza, para la diferencia de medias de ambos promedios escolares, se obtiene un valor de $\operatorname{Pr}(\mathrm{T}>\mathrm{t})=0,2757$. Al comparar estadísticamente los resultados del pre-test de ambos grupos, se tiene un valor de $\operatorname{Pr}(\mathrm{T}>\mathrm{t})=0,835692$.

Estos resultados permiten determinar que ambos grupos no presentan diferencias significativas, por lo que se puede determinar que ambos grupos son comparables y pertenecen a una misma población.

\section{Aplicación y Metodología}

Esta investigación se llevó a cabo en 3 semanas, donde cada grupo tuvo 12 sesiones de 45 minutos cada una, organizadas en 6 módulos de 90 minutos. Dentro de estos módulos se incluye el pre-test y el pos-test.

Ambos grupos (control y experimental) se sometieron a un pre-test y un pos-test antes de iniciar la intervención y una vez finalizado el proceso. Ambos test corresponden a una prueba de producción escrita individual. Se consideró la producción individual en ambas etapas, ya que el propósito es identificar de qué manera los estudiantes desarrollan sus habilidades personales en la producción escrita con un trabajo colaborativo.

\section{Los instrumentos de evaluación}

Para medir los resultados del proceso de intervención lingüística se creó un test, el que incluye 3 habilidades lingüísticas:

1. Reconocer el tema principal de un texto. (5 minutos): Para dar cuenta de esta actividad, el alumno leyó un texto informativo y en una oración mencionó el tema de este texto.

2. Identificar las ideas principales de una lectura. (20 minutos): Para ello, se le otorgó un espacio equivalente al número de ideas principales existentes, que para este caso, fueron 7. Incluso se enumeraron los espacios, de modo que el alumno supiera de ante mano la cantidad de ideas principales que debe identificar.

3. Producir un resumen de un texto. (20 minutos): El alumno utilizó las ideas principales que extrajo del texto para producir un resumen. No se preguntó explícitamente sobre los conectores, por cuanto se evaluó el uso de ellos, sin mediar intervención en el pre-test. 
Con el fin de enmascaramiento, se llamó Evaluación Formativa, ya que los alumnos estaban familiarizados con esta terminología y realizaban este tipo de evaluaciones a diario en el establecimiento. La técnica de enmascaramiento es un procedimiento realizado con el fin de que los miembros del equipo investigador y/o los participantes de un estudio no conozcan hechos $u$ observaciones que pudieran ejercer un cambio en sus acciones o decisiones y sesgar los resultados (Fracchia, 2009). Existen varios tipos de enmascaramiento: simple o ciego simple, doble ciego y triple ciego. Para esta investigación se ha utilizado el enmascaramiento ciego simple, donde lo sujetos desconocen a qué grupo pertenecen, si de control o experimental. Además, desconocen que son partes de una investigación (Rada, 2007).

Para determinar la confiabilidad de los instrumentos, se analizaron ambos test utilizando el coeficiente Alfa de Cronbach. De acuerdo a esta medición, se obtuvo un resultado de 0,95. A partir de estos datos, podemos señalar que los instrumentos utilizados en la intervención mantienen una consistencia interna de los ítems analizados.

Para determinar la validez de los instrumentos, se llevó a cabo una evaluación de expertos, la que estuvo formada por el jefe de la Unidad Técnico Pedagógica y la Coordinadora de Lenguaje del establecimiento en el cual se realizó la intervención.

\section{Diseño del módulo de intervención}

En adelante, se explica el módulo de intervención lingüística según la teoría de Hubbard.

- Módulo de desarrollo:

El enfoque: En esta investigación se trabajó en función de un enfoque metodológico basado en el Enfoque por Tareas y el Enfoque Colaborativo utilizando herramientas web 2.0 en un diseño CALL. En este estudio estos elementos determinarán si el diseño CALL es pertinente para generar aprendizaje de un tipo de reducción de textos.

El diseño: El objetivo lingüístico de esta intervención fue la producción de un resumen. Las metas de aprendizaje fueron: el aprendizaje de la habilidad lingüística de producir resúmenes y la capacidad de comunicar un resumen a un nivel público vía web. El plan de trabajo se llevó a cabo en 12 sesiones, para las cuales hubo tareas definidas. 
El Procedimiento: El tipo de actividad que los alumnos desarrollaron en esta intervención fue la producción colaborativa, en grupos de 2 estudiantes, de un resumen de un texto informativo. En cuanto a los materiales, fueron trabajados con herramientas online: Google Docs (formularios, presentaciones ppt) y Blog.

- Módulo de Implementación: Uno de los elementos a considerar es la accesibilidad: en relación a este aspecto importante, el establecimiento tenía una sala de computación con 22 computadores, uno cada dos estudiantes. Además tenía el soporte y el acceso necesarios para trabajar con herramientas online, ya que hubo un laboratorio óptimo para ello. Las actividades de seguimiento estuvieron determinadas por los archivos que Google Docs guardaba de manera automática, en los que se mencionan los cambios realizados, quién realizó los cambios, cuándo y a qué hora. Además fue guardando un historial de trabajo de cada grupo.

- Modulo de Evaluación: Cabe destacar que los resúmenes se evaluaron mediante una prueba de producción escrita tanto en pre-test como en el pos-test. Los criterios para evaluar la prueba estuvieron determinados de acuerdo a la taxonomía propuesta, basada en los constructos teóricos de Cassany (2004).

\begin{tabular}{lll}
\hline \multicolumn{1}{c}{ Conector } & \multicolumn{1}{c}{ Función } & \multicolumn{1}{c}{ Ejemplos } \\
\hline Ordenadores & $\begin{array}{l}\text { Ordenan el } \\
\text { momento en el que } \\
\text { ocurren los hechos. }\end{array}$ & $\begin{array}{l}\text { En primer lugar/en segundo } \\
\text { lugar; por una parte/por otra } \\
\text { parte; de un lado/de otro lado, } \\
\text { actualmente, finalmente, etc. }\end{array}$ \\
\hline Consecutivos & $\begin{array}{l}\text { Indican la } \\
\text { consecuencia de un } \\
\text { hecho. }\end{array}$ & $\begin{array}{l}\text { En consecuencia, por tanto, } \\
\text { de modo que, por esto, a } \\
\text { consecuencia de, así que, por } \\
\text { lo cual pues por consiguiente, } \\
\text { razón por la cual, etc. }\end{array}$ \\
& $\begin{array}{l}\text { En cualquier caso, en todo caso, } \\
\text { de todos modos, etc. }\end{array}$ \\
& $\begin{array}{l}\text { Indican una } \\
\text { reformulación } \\
\text { para un hecho } \\
\text { determinado. }\end{array}$ & \\
\hline
\end{tabular}




\begin{tabular}{lll}
\hline Aditivos & Añaden información & $\begin{array}{l}\text { Por otra parte, además, encima, } \\
\text { aparte, incluso, etc. }\end{array}$ \\
\hline Adversativa & Indican una & No obstante en cambio, ahora \\
& oposición entre dos & bien, con todo, sin embargo \\
& ideas. & antes bien, por el contrario, por \\
& el contrario, de todas maneras. \\
\hline
\end{tabular}

Tabla 1. Taxonomía propuesta para el uso de marcadores textuales, basada en la Taxonomía de Cassany (2004).

En la Tabla 1 se muestra la taxonomía propuesta para el uso de marcadores textuales.

Cabe destacar que esta intervención se realizó durante las clases normales de la asignatura de Lenguaje y Comunicación. Esto permitió que se llevara a cabo de manera natural para los estudiantes.

\section{Análisis de los Resultados}

A continuación se presentan los resultados obtenidos en la prueba de producción escrita utilizada como pre-test y post-test tanto del Grupo Experimental como del Grupo de Control. Para evaluar esta prueba se consideró una rúbrica que contenía la taxonomía propuesta para la evaluación de los resúmenes.

El puntaje total de cada test fue 20 puntos. Se evaluaron 3 indicadores:

1. Reconocer el tema: Puntaje total 2.

2. Identificar ideas principales del texto: Puntaje total 14 (2 puntos como máximo por cada idea principal, las que en total eran 7).

3. Producción de un resumen: Puntaje total 4 (dentro de esta habilidad se evaluaba el uso de conectores para la unión de ideas principales).

En el Gráfico 1 se detalla el porcentaje de logro por habilidad que obtuvo el grupo de control en el pre-test y en el post-test. 


\section{GRUPO DE CONTROL}

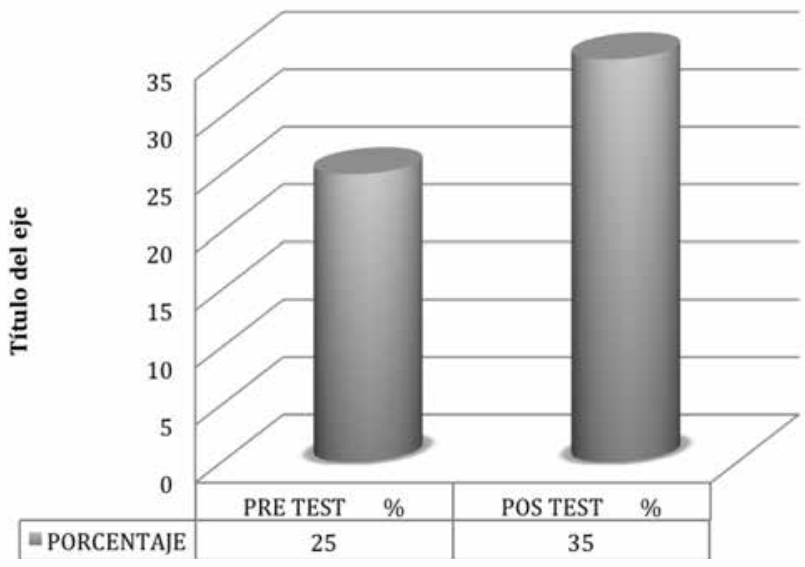

Gráfico 1. Resultados del pre-test y post-test del grupo de control en porcentaje de logro.

De acuerdo con esta información, se puede concluir que el grupo de control avanzó un 10\% de logro entre cada test.

Por su parte, en el Gráfico 2 se puede apreciar los porcentajes de logro alcanzados por el grupo experimental tanto en el pre como en el posttest. Este grupo logró avanzar en un $28 \%$ más entre cada test.

\section{GRUP0 EXPERIMENTAL}

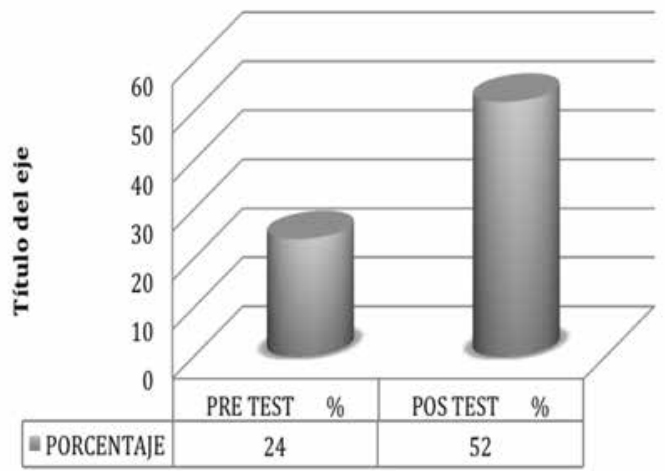

Gráfico 2. Resultado del pre-test y post-test del grupo experimental en porcentaje de logro. 
Al comparar los resultados obtenidos en el pre-test y post-test por cada uno de los grupos, se observa que ambos avanzaron en cuanto a los porcentajes; sin embargo, el grupo experimental ostenta una mayor diferencia que el grupo control. En el Gráfico 3, se puede observar que no hay diferencia entre los porcentajes de logro obtenidos por ambos grupos en el pre-test. En el post-test, es posible apreciar una diferencia sustancial, ya que el grupo experimental logró $17 \%$ de logro más que el del grupo control.

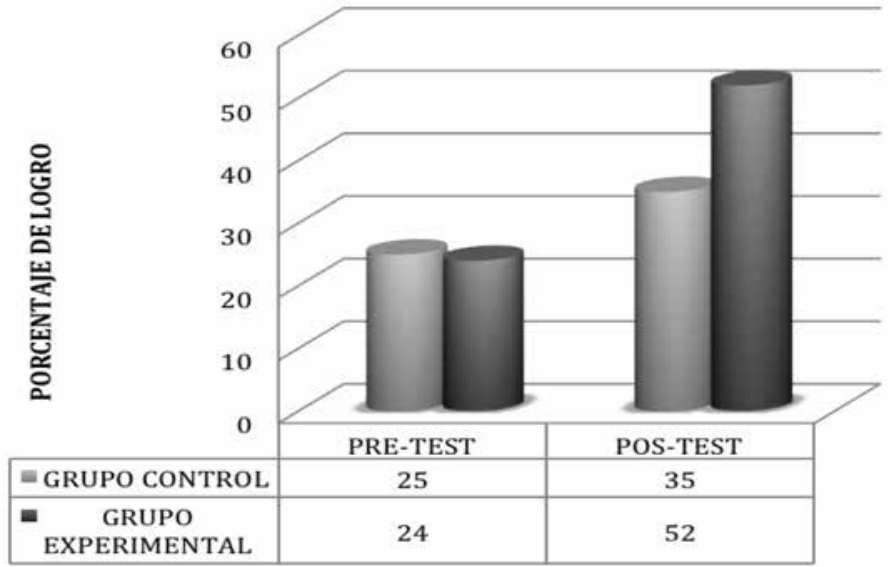

Gráfico 3. Comparación de los porcentajes de logro obtenidos por el grupo experimental y el grupo control en pre-test y post-test.

En cuanto a las habilidades, el Gráfico 4 muestra que la habilidad de "producción de resumen" fue la que arrojó una mayor diferencia de resultados entre los grupos experimental y de control. Esto evidencia que el grupo experimental obtuvo mejores resultados. A su vez, se puede apreciar que la habilidad menos desarrollada en ambos grupos fue el reconocimiento del tema del texto informativo. Se demuestra que ni el grupo experimental ni el de control lograron desarrollar esta habilidad. Ambos grupos tienen 0,4 punto (lo que se traduce en un 1\% de logro), siendo el máximo 2. 


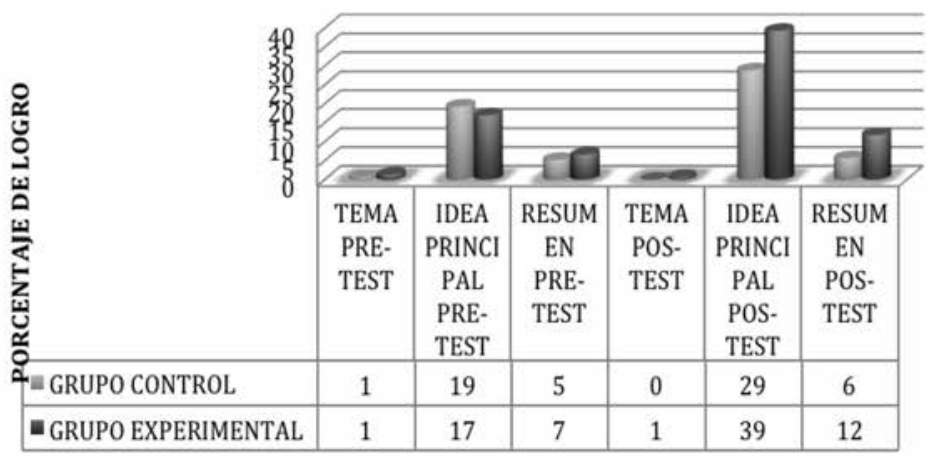

Gráfico 4: Comparación de los porcentajes de logro por habilidad obtenidos por el grupo experimental y el grupo control en pre-test y post-test.

En ambos grupos podemos evidenciar que de las tres habilidades evaluadas, reconocer un tema, identificar ideas principales y producir un resumen, la habilidad de producir un resumen fue la que obtuvo un mayor incremento, entre el pre y pos-test de ambos grupos. El identificar ideas principales, si bien no tuvo los resultados que se esperaban, logró un avance con respecto a los resultados obtenidos en el pre-test. La habilidad menos desarrollada fue la reconocer el tema, ya que muy pocos alumnos lograron responder esa pregunta correctamente y, así, dar cuenta de su capacidad de síntesis. Por lo mismo, en los resultados aparece que esta habilidad tiene solo $1 \%$ de logro.

Cabe destacar que la habilidad de reconocer el tema no fue desarrollada de forma significativa, ya que el foco de la investigación estaba en el uso de conectores para la unión de idas principales en la producción del resumen.

Como se puede observar en el punto anterior y en los gráficos precedentes, la distribución de los resultados del pre-test del grupo de control y del grupo experimental son similares: $25 \%$ de logro cada uno respectivamente. No obstante, se considera pertinente comprobar su validez estadística por medio de una prueba $T$ Student para la comparación de las medias de ambos grupos, donde se debe probar una de las dos hipótesis dadas de $\mathrm{H} 0$ : diferencia=0, y H1: diferencia $>0$, donde la diferencia es la media de las diferencias, observación por 
observación, entre los valores del promedio de dos grupos. Se utiliza este tipo de estadístico, ya que la investigación considera un tamaño muestral distribuido en dos grupos, y además se aplica a una población que sigue una distribución normal. Esta prueba fue aplicada con un nivel de confianza del $95 \%$. Estos resultados son corroborados por el programa STATA 11.

A continuación se procederá a comparar los resultados en el pre y pos-test del grupo experimental.

Se establece formalmente la hipótesis de trabajo para esta prueba:

H1: Existe una diferencia significativa entre los resultados del pre-test y post-test del grupo experimental.

H0: No existe una diferencia significativa entre los resultados del pre-test y post-test del grupo experimental.

Este estadístico distribuye $T$ student con $n$-1 grado de libertad. Ahora bien, la probabilidad del estadístico resultante de la hipótesis es $t_{c}=5,5120$, y la $\operatorname{Pr}(T>t)=0,0000$. Lo que se determina primero es que la media de la diferencia entre el post y pre-test es mayor a cero, ello quiere decir que los valores del post-test son mayores que los valores del pre-test en promedio. A partir de estos resultados se rechaza la $\mathrm{H0}$ y podríamos concluir que los valores del post-test son significativamente mayores que los del pre-test.

A continuación se muestra la Tabla 1 con el análisis realizado para la comparación entre el pre-test y post-test del grupo experimental.

\begin{tabular}{|c|c|c|c|c|c|c|}
\hline variable & obs & Mean & std. Err. & std. Dev. & [95\% conf. & Interval] \\
\hline $\begin{array}{l}\text { posttest } \\
\text { pretest }\end{array}$ & $\begin{array}{l}22 \\
22\end{array}$ & $\begin{array}{l}10.36364 \\
4.772727\end{array}$ & $\begin{array}{l}1.043524 \\
.7783094\end{array}$ & $\begin{array}{l}4.894559 \\
3.650594\end{array}$ & $\begin{array}{r}8.19351 \\
3.154144\end{array}$ & $\begin{array}{r}12.53376 \\
6.39131\end{array}$ \\
\hline diff & 22 & 5.590909 & 1.014311 & 4.757541 & 3.481533 & 7.700285 \\
\hline \multicolumn{2}{|c|}{$\begin{array}{r}\operatorname{mean}(\text { diff })= \\
\text { o: } \operatorname{mean}(\text { diff })=\end{array}$} & $n$ (posttest & pretest) & degrees & of freedom & $\begin{array}{r}5120 \\
21\end{array}$ \\
\hline \multicolumn{2}{|c|}{$\begin{array}{l}\text { Ha: } \operatorname{mean}(\text { diff })<0 \\
\operatorname{Pr}(T<t)=\mathbf{1 . 0 0 0 0}\end{array}$} & Pr ${ }^{H a}$ & $\begin{array}{l}\operatorname{mean}(\text { diff }) \\
|>| t \mid)=\end{array}$ & $\begin{array}{l}!=0 \\
0000\end{array}$ & \multicolumn{2}{|c|}{$\begin{array}{l}\text { Ha: mean(diff) }>0 \\
\operatorname{Pr}(T>t)=0.0000\end{array}$} \\
\hline
\end{tabular}

Tabla 1. Resultados obtenidos mediante el estadístico STATA 11 para comparar los resultados del pre-test y pos-test del grupo experimental.

Conclusión: se acepta como verdadera H1 y se rechaza H0. 
A continuación se procederá a comparar los resultados entre el pretest y el pos-test del grupo de control.

Se establece formalmente la hipótesis de trabajo para esta prueba:

H1: Existe una diferencia significativa entre los resultados del pre-test y pos-test del grupo de control.

H0: No existe una diferencia significativa entre los resultados del pre-test y pos-test del grupo de control.

La Tabla 2 muestra la comparación de resultados entre el pre y el pos-test del grupo de control. Al obtener la probabilidad del estadístico $\mathrm{t}=-2.8005$, la $\operatorname{Pr}(\mathrm{T}<\mathrm{t})=0$,9946. Si bien, a partir de estos resultados podemos apreciar que el grupo de control aprendió, al ser mayor que 0,05 significa que la diferencia entre el pre-test y el post-test no es significativa.

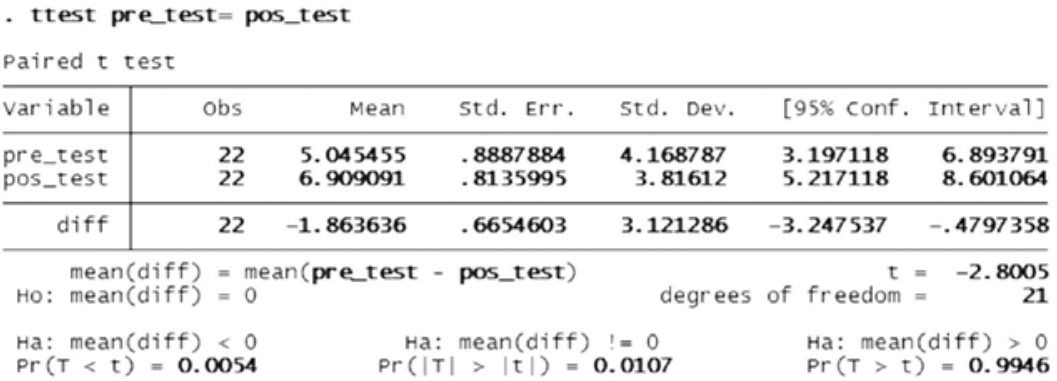

Tabla 2. Resultados obtenidos para comparar los resultados del pre y post-test del grupo control.

Por lo tanto, se rechaza H1 y se acepta como verdadera H0.

De este modo, podríamos determinar que los valores del post-test son significativamente mayores que los del pre-test comparando al grupo de control con el grupo experimental y, por lo tanto, los resultados del grupo experimental son estadísticamente superiores en relación al grupo de control.

Por ende, se acepta como verdadera la hipótesis de esta investigación: Un diseño CALL que combine el enfoque por tareas y el enfoque colaborativo utilizando herramientas web 2.0 mejora la producción de resúmenes de textos informativos en estudiantes de sexto básico. 


\section{Conclusiones}

El resumen es un tipo de reducción de textos que da cuenta de la comprensión textual de una lectura; es por ello que resulta indispensable buscar estrategias que les permitan a los alumnos aprender esta habilidad de forma efectiva, considerando que se desarrolla en todos los niveles académicos.

A la luz de los resultados obtenidos, concordamos con lo que plantea García y Ferreira (2010) ya que se ha obtenido evidencia que el uso de una aplicación computacional incrementa el aprendizaje de un aspecto lingüístico de la lengua materna. En este caso, el aprendizaje que se desarrolló fue la producción de resúmenes.

Hay que considerar entonces que el entorno de aprendizaje electrónico reunió características que fueron realmente poderosas para la colaboración, tales como su interactividad, ubicuidad, y sincronismo. Los alumnos que trabajaron colaborativamente comprobaron que existe un nuevo contexto sociocultural, el que define que aprenden mejor socialmente en red, que de forma individual (Zañartu, 2008).

Se confirma lo dicho por Chapelle (2001) cuando menciona que actualmente se incorpora en el aprendizaje del alumno un nuevo conjunto de prácticas, lo que les permite aumentar sus conocimientos, tal y como se puede observar en los resultados.

Los computadores, de forma aislada como ocurrió en el grupo de control, ofrecen una cantidad de posibilidades de aprendizaje, pero conectados, como en el grupo experimental incrementaron su funcionalidad en varios órdenes de magnitud (Adell, 1997). Esto se demuestra la diferencia en los resultados entre ambos grupos. Tanto el grupo de control como el grupo experimental trabajaron con computadores, pero claramente el grupo experimental tuvo un uso más efectivo.

A la luz de los resultados obtenidos se concluye que el diseño utilizado mejora el desarrollo de la producción de resúmenes. El grupo experimental demostró tener resultados muy superiores al grupo de control. Si bien ninguno de los dos grupos demostró superar el $60 \%$ de logro en el pre-test y post-test, es posible señalar que el grupo experimental obtuvo resultados que permiten concluir que tuvieron un aprendizaje significativo en cuanto a la producción de resúmenes. 
En cuanto al diseño CALL, se puede señalar que su implementación les permitió a los estudiantes trabajar de forma más autónoma y responsable en relación a su aprendizaje, dejando claro los roles que tienen, tanto los alumnos como los docentes, hoy en día en la era digital. El avance de la tecnología ha puesto mayores desafíos en el quehacer docente, los que deben llevarse a cabo de forma efectiva si se espera desarrollar habilidades permanentes en los estudiantes.

Para este nuevo escenario educativo, queda demostrado que un diseño CALL es pertinente, ya que permite abarcar todas las complejidades del proceso de aprendizaje asistido por computador.

El uso de los Enfoques por Tarea y Colaborativo, potenciaron un trabajo de aula autónomo y generaron oportunidades de interacción constante para alcanzar la tarea de cada sesión.

Queda en evidencia que el uso de las herramientas seleccionadas de la web 2.0, claramente, fortalecen la escritura en colaboración y permite, a los alumnos, trabajar sin temor al error.

Finalmente, cabe destacar que el aporte de esta investigación al ámbito de la Lingüística Aplicada radica en que provee un diseño metodológico efectivo para el aprendizaje del resumen en la lengua materna. 


\section{Referencias bibliográficas}

Adell, J. (1997). Tendencias en educación en la sociedad de las tecnologías de la información. Revista Electronica de Tecnologia Educativa. Recuperado de <http://www.uib.es/ depart/gte/ edutec-e/ revelec7 / revelec7.html>

Bustamante, G. y Jurado, F. (Comp.) (1997). Entre la lectura y la escritura: hacia la producción interactiva de los sentidos. Santa Fe de Bogotá: Magisterio.

Calzadilla, M. E. (2001). Aprendizaje Colaborativo y Tecnologías de la información y la comunicación. Recuperado de <http://www.gfbnm.com/ibanks/pub/ CAIintraDemo/AprendizajeColaborativoArticulo/ AprenColabyTecInfCom.pdf $>$

Cameron, K. (ed.). (1999). CALL: Media, Design \& Applications. The Netherlands: Swets \& Zeitlinger Publishers.

Cassany, D. (2004). La cocina de la escritura. Barcelona, España: Anagrama. Chapelle, C. (2001). Computer Applications in Second Language Acquisition. Foundations for teaching, testing and research. Cambridge: Cambridge University Press.

Condemarín, M. y Medina, A. (1998). Lenguaje integrado II: desarrollo de las competencias lingüísticas y comunicativas de los estudiantes de $5^{\circ}$ a $8^{\circ}$ año de EGB: Fundamentos y estrategias. Santiago, Chile: Ministerio de Educación.

Egbert, J. \& Mikel, G. (2005). CALL Research Perspectives. New Jersey: Lawrence Erlbaum Associates, Inc.

Estaire, S. (2007). La Enseñanza de Lenguas mediante Tareas: principios y planificación de unidades didácticas. Recuperado de $<$ http: / / ulises.cepgranada.org/moodle/pluginfile. php / 25786/mod_page/ content / 1/docs / tareas_ Sheila.pdf $>$

Fracchia, L. (2009). El Diseño Metodológico: Tipos de Investigación. Recuperado de <http://www.scemt.com.ar/ Met09/Disenos.pdf> 
García, J. \& Ferreira, A. (2010). Entrenamiento en estrategias de aprendizaje de inglés como lengua extranjera en un contexto de aprendizaje combinado. Revista Nebrija de Lingüística Aplicada, 8 (4), 17- 4.

Gokhale, A. (1995). Collaborative Learning Enhances critical thinking. Journal of Technology Education, 7(1), 22-30.

Gruba, P. (2004). Computer Assisted Language Learning (CALL). En The Handbook of Applied Linguistic (pp. 623-649). Blackwell Publishing Ltd. United Kingdom.

Hubbard, P. (1996). Elements of CALL methodology: development, evaluation, and implementation. The power of CALL (pp. 15-32). Houston: Athelstan Publications.

(Ed.). (2009). Computer Assisted Language Learning: Critical Concepts in Linguistics, Volumes I-IV. London \& New York: Routledge.

Lázaro, O. (2009). Web 2.0, comunicación y material didáctico digital para el aprendizaje del español: el aula virtual de español del instituto cervantes y su actualización. Revista de Lingüística Teórica y Aplicada. 47 (2), II Sem., 13-34.

Levy, M. \& Stockwell, G. (2006). CALL dimensions, Options and Issues in CALL. New Jersey: Laurence Erlbaum Associates.

Long, M. (1985). The Design of Classroom Second Language Acquisition: towards Task-based Language Teaching. En Hyltenstam, K and Pienemann, M (Eds.), Modelling and assessing second language acquisition (pp. 77-99). Clevedon: Multilingual Matters.

Nunan, D. (2001). Aspects of Task-Based Syllabus Design. Hong Kong: The English Centre, University of Hong Kong.

Pennington, M. (2004). Electronic media in second language writing: An overview of tools and research findings. En Fotos. S. \&. Browne C. M (Eds.), New perspectives on CALL for second language classrooms (pp. 69-92). Mahwah, NJ: Erlbaum. 
Piscitelli, A. (2010). Segunda Parte. Educando a los nativos digitales en espaciosdeafinidad.Nativosdigitales-ellibro/elweblog: dieta cognitiva, inteligencia colectiva y arquitecturas de la participación. Recuperado de <http://www. nativos-digitales.com.ar $/ \mathrm{p}=8 \#$ more- $8>$

Rada, G. (2007). El ensayo clínico controlado (ECC). Recuperado de 2013 de <http://escuela.med.puc.cl/Recursos/ recepidem/PDF/estExper03.pdf>

Richards, J. \& Rodgers, T. (2004). Approaches and methods in language teaching. Cambridge: Cambridge University Press.

Skehan, Peter (2008). A Cognitive Approach to Language Learning. Oxford: Oxford University Press.

Solé, I. (1996). Estrategias de lectura. Barcelona: Institut de Ciencias de l'Education.

Venegas, R. (2010). Evaluación de resúmenes de textos narrativos y expositivos utilizando analisis semántico latente. Revista Signos, 44 (75), 85-102. Recuperado de <http: / / www.scielo.org.ar/pdf/spc/v14n2/ v14n2a26.pdf $>$

Willis, D. \& Willis, J. (2007). Doing task based teaching. Oxford: Oxford University Press.

Zañartu, L. (2008). Aprendizaje colaborativo: una nueva forma de diálogo interpersonal y en red. Educar Chile. Recuperado de <http:/ / www.educarchile.cl/portal.base/web / vercontenido.aspx?id=139860> 
